\title{
Heat source/sink and Newtonian heating effects on convective micropolar fluid flow over a stretching/shrinking sheet with slip flow model
}

\author{
Muhammad Kamran \\ Department of Mathematics and Statistics, Curtin University of Technology, GPO Box U1987, Perth WA 6845 Australia
}

Corresponding Author Email: muhammad.kamran@curtin.edu.au

https://doi.org/10.18280/ijht.360212

Received: 1 November 2017

Accepted: 28 April 2018

\section{Keywords:}

micropolar fluid, stretching/shrinking sheet, slip flow model, Newtonian heating

\begin{abstract}
The purpose of this study is to analyse the combined effects of the heat generation(source)/ absorption (sink) and Newtonian heating on the mixed convective micropolar fluid flow past over a stretching/shrinking porous sheet. Slip flow model is also taken into account in this investigation. The governing flow behaviour is designed by coupled partial differential equations and then transformed into a system of coupled nonlinear ordinary differential equations with the mixed derivative boundary conditions. A semi-analytical approach named Homotopy Analysis Method (HAM) is applied to solve this transformed system of nonlinear equations. Influences of the pertinent dimensionless parameters on the prescribed velocities and temperature profiles along with the physical quantities are presented in the graphical and tabular illustrations. For special cases, it is found that the obtained solutions are excellent in agreement with the available results. In this study, it is observed that when the sheet stretches or shrinks, the temperature of the micropolar fluid flow increases with an increase in the heat generation and Newtonian heating parameters and it decreases with an increase in the heat absorption parameter and the Prandtl number. This investigation of the heat generation and absorption on the micropolar fluid flow with slip flow effects has shown the useful information which could be helpful for crystal growing in the industry and the processes to polish the artificial heart valves and the internal cavities.
\end{abstract}

\section{INTRODUCTION}

Micropolar fluids belong to a subclass of simple microfluids with non-symmetric stress tensor and present the microrotational inertia and micro-rotational effects. Geometrically, they consist of randomly oriented rigid spherical microparticles suspended in a viscous medium where the deformation of these particles is not considered. The physical properties and behaviour of this viscous medium are affected by the prescribed micro-particles contained in each of its volume element. The theory of simple microfluids and micropolar fluids was proposed by Eringen [1-2]. Various concepts, which are not given in the classic fluid mechanics, were introduced including the inertial spin, body moments, micro-stress averages and stress moments. Eringen [3] later extended the theory to thermo micropolar fluids to study the heat conduction and heat dissipation effects and moreover, reported other aspects in [4]. Detailed treatises on various features of micropolar fluids were given by Lukaszewicz [5].

In the past few decades, the flow of micropolar fluid past over a stretching sheet is under investigation because of its numerous applications in industry, for example, paper production, crystal growing, tinning and annealing of metals. Kelson and Farrell [6] examined the Suction and injection effects on the porous stretching sheet. The effect of surface condition on the micropolar fluid flows past over a porous stretching surface has been studied by Kelson and Desseaux [7]. Bhargava et al. [8] extended their work to the mixed convective incompressible micropolar fluid flow by using a numerical method. Heat source/sink effect of water-based nanofluid flow through a permeable stretching tube has been analysed by Ahmed et al. [9]. Chand et al. [10] investigated the Brownian diffusion and the thermophoresis effects on the horizontal layer of the nanofluid. Exact analytical solution for the heat transfer micropolar fluid flow over permeable shrinking/stretching sheets was reported by Turkyilmazoglu [11-12]. The influence of the thermal radiation on the nano magneto-micropolar fluid flow over a permeable stretching sheet with a non-uniform heat source/sink was numerically investigated by Pal and Mandal [13].

In many engineering applications, it has been observed that the fluid particles close to the solid surface slip along the surface due to their finite tangential velocity. This flow regime is termed as a slip-flow regime in which shear stress depends on the slip velocity. This effect cannot be ignored. The study of the slip-flow regime has many technological applications, e.g. refrigerating coils, transmission lines, polishing the artificial heart valves and internal cavities. Wu [14] derived a slip flow model with the help of the kinetic theory for arbitrary Knudsen number. He found that it is a preferable approach to investigate the slip flow behaviour other than that of the Maxwell slip model [15], second-order slip model [16], 1.5 $5^{\text {th }}$ order slip model [17] and Fukui-Kaneko slip model [18]. Recently, Wu's slip flow model has been used in many investigations of the Newtonian [19-22] and non-Newtonian such as micropolar [23-26] fluid flows over different geometries.

However, in comparison with the already published work, this study has two new features. Firstly, the flow behaviour considering the effects of the heat generation and absorption 
at the weak concentration of microelements. Secondly, the Newtonian heating effect on the mixed convective micropolar fluid flow over the stretching/shrinking permeable sheet with the second order slip flow model is tackled which, to the best of our knowledge, has never been investigated before.

\section{GOVERNING EQUATIONS}

Consider a steady state incompressible micropolar fluid flow over a vertical permeable stretching/shrinking sheet with velocity $u=u_{w}(x)$, where $u_{w}(x)=a x$ along the $x$-axis direction and $a>0$, in addition to the slip flow model introduced by $\mathrm{Wu}$ [14]. The flow is taken along the $x$-axis and the $y$-axis is normal to the surface, as shown in Figure 1. The acceleration due to gravity $(g)$ is acted in the downward direction and Newtonian heating [27] is introduced in the thermal boundary condition. Under thermal boundary layer and the usual Boussinesq approximations, the governing system of equations [23]

$\frac{\partial u}{\partial x}+\frac{\partial v}{\partial y}=0$

$u \frac{\partial u}{\partial x}+v \frac{\partial u}{\partial y}=\frac{\mu+\chi}{\rho}\left(\frac{\partial^{2} u}{\partial y^{2}}\right)+g \beta_{T}\left(T-T_{\infty}\right)+\frac{\chi}{\rho}\left(\frac{\partial \omega}{\partial y}\right)$,

$u \frac{\partial \omega}{\partial x}+v \frac{\partial \omega}{\partial y}=\frac{\gamma}{\rho j}\left(\frac{\partial^{2} \omega}{\partial y^{2}}\right)-\frac{\chi}{\rho j}\left(2 \omega+\frac{\partial u}{\partial y}\right)$

$u \frac{\partial T}{\partial x}+v \frac{\partial T}{\partial y}=\frac{k}{\rho c_{p}}\left(\frac{\partial^{2} T}{\partial y^{2}}\right)+\frac{Q_{0}}{\rho c_{p}}\left(T-T_{\infty}\right)$

with the boundary conditions

$$
\begin{aligned}
& u=s u_{w}(x)+u_{\text {slip }}, \quad v=v_{0}, \quad \omega=-n \frac{\partial u}{\partial y}, \\
& \left.\begin{array}{c}
k \frac{\partial T}{\partial y}=-h_{s} T \text { at } y=0, \\
u \rightarrow 0, \quad \omega \rightarrow 0, \quad T \rightarrow T_{\infty} \text { as } y \rightarrow \infty
\end{array}\right\}
\end{aligned}
$$

where $u$ and $v$ are the velocities along the axis of flow and the normal to it, respectively. The spin gradient viscosity is considered as $\gamma=(\mu+\chi / 2) j=\mu(1+K / 2) j$ for $K=\chi / \mu$ and $j=v / a$ are the material parameter and microinertia density [28], respectively.

Moreover, $v_{0}>0$ is the suction and $v_{0}<0$ is the injection velocity of the permeable stretching/shrinking sheet. Furthermore, the constant $n$ has the rang $0 \leq n \leq 1$, in which $n=0$ represents the strong concentration of microelements near the sheet which are unable to rotate [10], $n=0.5$ shows the vanishing of the of antisymmetric stress tensor which depicts the weak concentration, and $n=1$ can be used for the turbulent flow. However, $n=0.5$ which corresponds to the dilute micropolar fluid and this dilute fluid is considered in the present study. Furthermore, $u_{\text {slip }}$ is slip velocity of the stretching $(s>0) /$ shrinking $(s<0)$ sheet, which is given by $\mathrm{Wu}[14]$ as

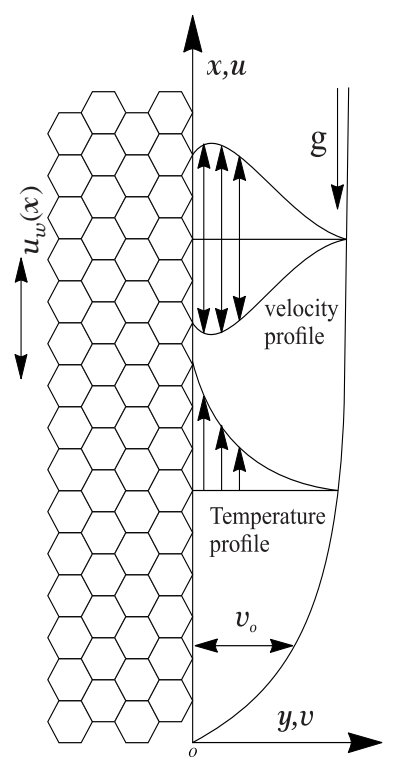

Figure 1. Flow model

$$
\begin{aligned}
u_{\text {slip }}= & \frac{2}{3}\left(\frac{3-\in l^{3}}{\in}-\frac{3}{2} \frac{1-l^{2}}{k_{n}}\right) d \frac{\partial u}{\partial y}-\frac{1}{4}\left(l^{4}+\frac{2}{k_{n}^{2}}\left(1-l^{2}\right)\right) \\
& d^{2} \frac{\partial^{2} u}{\partial y^{2}}=A \frac{\partial u}{\partial y}+B \frac{\partial^{2} u}{\partial y^{2}},
\end{aligned}
$$

where the constant $l$ is chosen from $\min \left(1 / k_{n}, 1\right)$ which leads to $0<l \leq 1$ for any value of the Knudsen $\left(k_{n}\right)$ number and $\in$ is the momentum accommodation coefficient which is defined as $0<\in \leq 1$. Furthermore, the molecular mean free path $d$ is always positive and therefore, $B$ is negative in magnitude. However, introducing the following similarity variables with velocity components $u=\partial \psi / \partial y$ and $v=-\partial \psi / \partial x$ as follow,

$$
\eta=\sqrt{a / v} y, \psi=\sqrt{a v} x f(\eta), \omega=\sqrt{a / v} \operatorname{axh}(\eta), \theta(\eta)=\frac{T-T_{\infty}}{T_{\infty}}
$$

where the stream function $(\psi)$ usually satisfies the equation of the conservation of mass $\mathrm{Eq}(1)$, and governing equations $(\mathrm{Eq}(2)-(4))$ are transformed into a set of non-linear coupled ordinary differential equations as follow,

$$
\begin{aligned}
& (1+K) f^{\prime \prime \prime}-\left(f^{\prime}\right)^{2}+f f^{\prime \prime}+\lambda \theta+K h^{\prime}=0, \\
& (1+K / 2) h^{\prime \prime}-h f^{\prime}+h^{\prime} f-K\left(2 h+f^{\prime \prime}\right)=0, \\
& \theta^{\prime \prime}+\operatorname{Pr} f \theta^{\prime}+Q \operatorname{Pr} \theta=0,
\end{aligned}
$$

with the boundary conditions

$$
\begin{aligned}
& f=f_{w,} \quad f^{\prime}=s+\alpha f^{\prime \prime}+\beta f^{\prime \prime \prime}, \\
& h=-n f^{\prime \prime}, \quad \theta^{\prime}=-\delta(1+\theta) \text { at } \eta=0 \text {, } \\
& f^{\prime} \rightarrow 0, \quad h \rightarrow 0, \quad \theta \rightarrow 0 \text { as } \quad \eta \rightarrow \infty,
\end{aligned}
$$


where the prime represents the derivative with respect to $\eta$. The non-dimensional physical parameters are defined in the form of the buoyancy parameter $\lambda=G r_{x} / \operatorname{Re}_{x}$ for the local Grashof number $G r_{x}=g \beta_{T} T_{\infty} x / a v$ and local Reynolds number $\operatorname{Re}_{x}=a x^{2} / v$, heat generation $(>0)$ or the heat absorption $(<0)$ parameter $Q=Q_{0} / a \rho c_{p}$ and Prandtl number $\operatorname{Pr}=\mu c_{p} / k$ in $\operatorname{Eq}(6)-(8) . \operatorname{In} \operatorname{Eq}(9), f_{w}=-(a v)^{-1 / 2} v_{0}$ is the suction $(>0)$ or injection $(<0)$ parameter, $\alpha=A \sqrt{a / v}>0$ and $\beta=B a / v<0$ are the first and second order slip flow parameters and $\delta=\sqrt{v / a} h_{s} / k$ is the Newtonian heating parameter. Moreover, the physical quantities in the form of the local skin friction coefficient $\left(C_{f x}\right)$, the local wall couple stress $\left(M_{x}\right)$ and the local Nusselt number $\left(N u_{x}\right)$ are expressed as

$$
\begin{aligned}
& C_{f x}=\frac{-\tau_{w x}}{\rho\left(u_{w}(x)\right)^{2}} \text { for } \tau_{w x}=(\mu+\chi) \frac{\partial u}{\partial y}+\left.\chi \omega\right|_{y=0}, \\
& M_{x}=\frac{-m_{w}}{\rho x\left(u_{w}(x)\right)^{2}} \text { for } m_{w}=\left.(\mu+\chi / 2) j \frac{\partial \omega}{\partial y}\right|_{y=0}, \\
& N u_{x}=\frac{-x q_{w}}{k_{f}\left(T-T_{\infty}\right)} \text { for } q_{w}=\left.k_{f} \frac{\partial T}{\partial y}\right|_{y=0},
\end{aligned}
$$

and the similarity transformation yields them

$$
\begin{aligned}
& C_{f x} \operatorname{Re}_{x}^{1 / 2}=-\left.(1+K-n K) f^{\prime \prime}\right|_{\eta=0}, \\
& M_{x} \operatorname{Re}_{x}=-\left.(1+K / 2) h^{\prime}\right|_{\eta=0}, \\
& N u_{x} \operatorname{Re}_{x}^{-1 / 2}=\left.\delta(1+1 / \theta)\right|_{\eta=0} .
\end{aligned}
$$

\section{SERIES SOLUTION}

A semi-analytical technique named Homotopy Analysis Method (HAM), introduced by Liao [29] is applied to solve the set of transformed nonlinear $\operatorname{Eq(6)-(8)~along~with~the~}$ boundary conditions $\mathrm{Eq}(9)$. HAM has a great advantage to control the convergence of the obtained series solutions and preferable to apply on the nonlinear ordinary differential equations as compared to the perturbation and nonperturbation, Adomian decomposition method, artificial small parameter method and $\delta$-expansion method (see reference in [29]). The basic features of the HAM solution in accordance with the nonlinear system of $\mathrm{Eq}(6)-(8)$ with corresponding boundary conditions $\operatorname{Eq}(9)$ are presented here.

The homotopy series solution for the $f(\eta), h(\eta)$ and $\theta(\eta)$ are expressed by a set of base functions $\left\{\eta^{p} e^{-q n} \mid p \geq 0, q \geq 0\right\}$ in the form of

$$
f(\eta)=\sum_{p=0}^{\infty} \sum_{q=0}^{\infty} a_{p, q} \eta^{p} e^{-q n}
$$

$$
\begin{aligned}
& h(\eta)=\sum_{p=0}^{\infty} \sum_{q=0}^{\infty} b_{p, q} \eta^{p} e^{-q n}, \\
& \theta(\eta)=\sum_{p=0}^{\infty} \sum_{q=0}^{\infty} c_{p, q} \eta^{p} e^{-q n},
\end{aligned}
$$

where $a_{p, q}, b_{p, q}$ and $c_{p, q}$ are constants. The first rule of solution expressions and boundary conditions yield the initial approximation for $\mathrm{Eq}(10)-(12)$ as

$f_{0}(\eta)=f_{w}+\frac{s\left(1-e^{-\eta}\right)}{1+\alpha-\beta}$

$h_{0}(\eta)=\frac{s n e^{-\eta}}{1+\alpha-\beta}$

$\theta_{0}(\eta)=\frac{\delta e^{-\eta}}{1-\delta} ; \delta \neq 1$

with the corresponding linear operators

$\mathcal{L}_{f}=\frac{d^{3}}{d \eta^{3}}-\frac{d}{d \eta}, \quad \mathcal{L}_{h}=\frac{d^{2}}{d \eta^{2}}+\frac{d}{d \eta}, \quad \mathcal{L}_{\theta}=\frac{d^{2}}{d \eta^{2}}+\frac{d}{d \eta}$

satisfying properties (as reported in [29, 30]) such as $\mathcal{L}_{f}\left(F_{1}+F_{2} e^{\eta}+F_{3} e^{-\eta}\right)=0, \mathcal{L}_{h}\left(F_{4}+F_{5} e^{-\eta}\right)=0$ and $\mathcal{L}_{h}\left(F_{6}+F_{7} e^{-\eta}\right)=0$ in which $F_{i}$ ( $i$ varies from 1 to 7 ) are the arbitrary constants. Moreover, auxiliary functions are defined as $H_{f}(\eta)=H_{h}(\eta)=H_{\theta}(\eta)=1$ and the auxiliary parameters are set as $\hbar_{f}=\hbar_{h}=\hbar_{\theta}=\hbar$ during the computation.

MATHEMATICA computation software is then used to solve the given system.

\subsection{Convergence control region}

The convergence of the Homotopy series solutions can be controlled by the non-zero auxiliary parameter $(\hbar)$, which is evident in Figure 2. For the sake of the validation of the current results and analysing the influence of the governing parameters on the prescribed velocities and temperature distributions, the appropriate values of $\hbar$ are chosen from the common tolerable range $-0.55 \leq \hbar<0$ which is obtained at the $20^{\text {th }}$-order of approximation of the HAM solution for $n=0.5, \operatorname{Pr}=5, K=\lambda=Q=\delta=f_{w}=0.1, \alpha=1$ and $\beta=-1$. Here, it is noticeable that when the sheet stretches $(s=1), \hbar$ curves are plotted at $\eta=0$, and when it shrinks, curves are sketched at $\eta=50$ to get the smooth horizontal lines. 


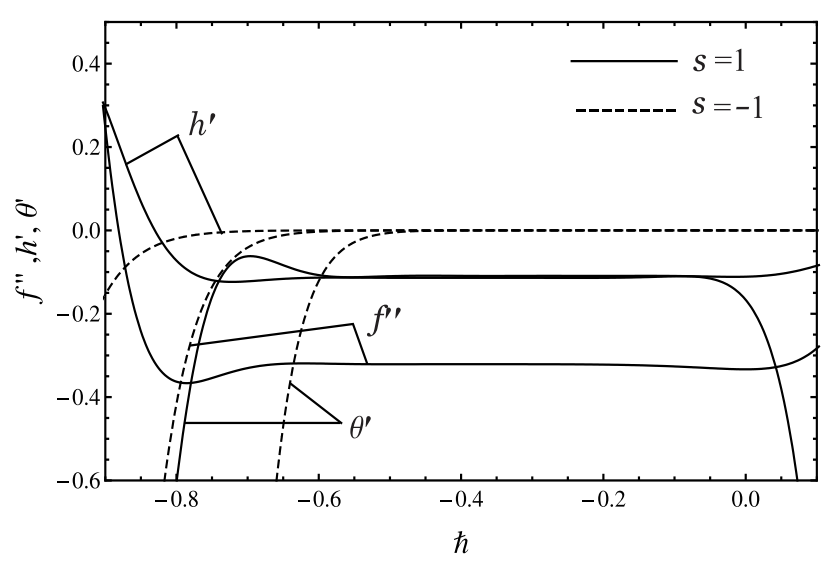

Figure 2. $\hbar$ curves

\subsection{Study validation}

The present study is validated in three different ways, firstly, the case of forced convective micropolar fluid flow over a porous shrinking sheet with the slip flow model as presented by Rosca and Pop [23] is considered. The obtained HAM solutions are in excellent agreements, as depicted in Figure 3 for the stable and physically reliable solutions as reported in [23] at $K=\alpha=1, f_{w}=3, s=\beta=-1, \lambda=0$ and $n=0.2$. In Figure 3 , the lower curve is sketched for velocity component $\left(f^{\prime}\right)$ at $\hbar=-0.095$ and an upper curve is plotted for microrotational velocity $(h)$ at $\hbar=-0.13$.

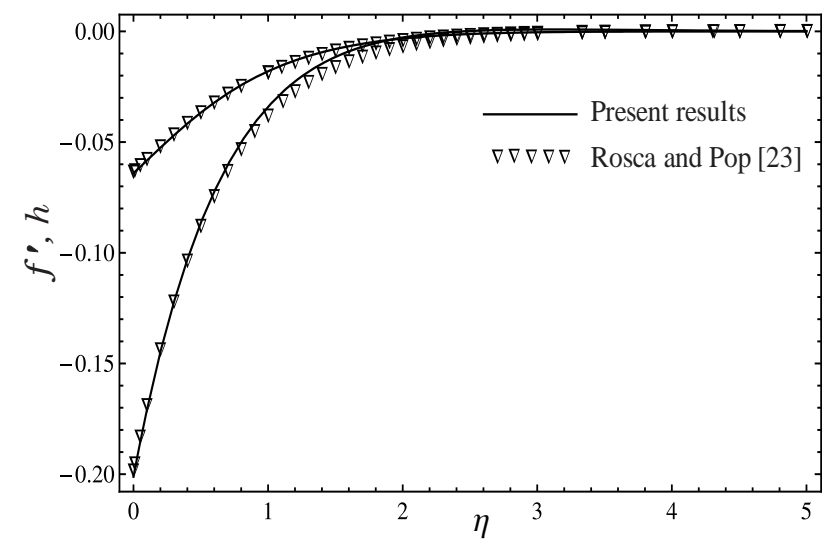

Figure 3. Validation with [23]

Secondly, the forced convective micropolar fluid flow over a permeable shrinking sheet [11] is validated with the current HAM results in a special case at $f_{w}=2.1, n=0.5, s=-1$, $K=0.1$ and $\lambda=\alpha=\beta=0$ as depicted in Figure 4, which is again in good correspondence. In Figure 4 , it is to be noted that the lower and upper curves are for $f^{\prime}$ and $h$, respectively, which are computed at $\hbar=-0.5$. Lastly, the present results are also in very good compatibility with the analytical results reported by Turkyilmazoglu [12] for the stretching sheet case at $K=1, f_{w}=s=1, \lambda=\alpha=\beta=0$ and $n=0.5$, as shown in Figure 5. The upper and lower curves are plotted for $f^{\prime}$ and $h$ at $\hbar=-0.25$ and -0.1 , respectively.

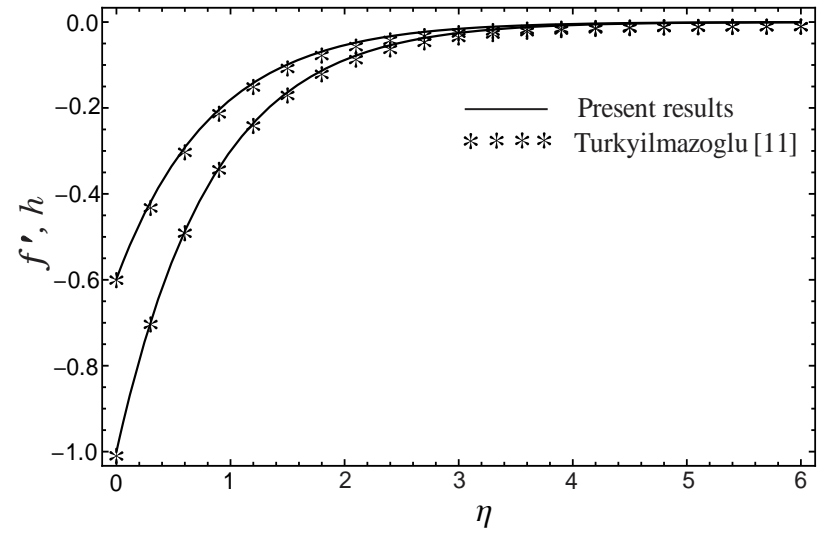

Figure 4. Validation with [11]

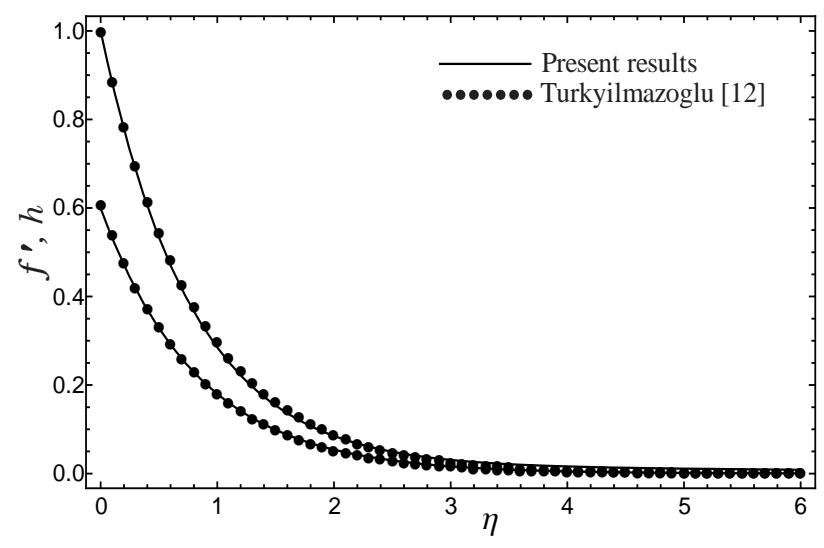

Figure 5. Validation with [12]

\section{RESULTS AND DISCUSSION}

In this section, obtained HAM results are discussed at the fixed values of the prescribed parameters $\beta=-1, \operatorname{Pr}=5$, $K=f_{w}=Q=\delta=n=0.5$ and $\lambda=\alpha=1$ for the stretching $(s=1)$ /shrinking $(s=-1)$ of the sheet or otherwise specified. Figures 6-7 illustrate the effect of the material parameter $K$ on the velocity component $\left(f^{\prime}\right)$ and the micro-rotational velocity (h) with the increasing value of $\eta$, respectively. It is observed that when the sheet stretches, with the increase of the concentration of microelements in the fluid flow, the prescribed velocities increase and generally, the Newtonian fluid $(K=0)$ has the lowest velocity as compared to the nonNewtonian, i.e. micropolar $(K>0)$ fluid flow. Moreover, when the sheet shrinks; the increasing concentration of microelements cause to slow down the velocity component $\left(f^{\prime}\right)$ and oppositely, enhance the micro- rotational velocity of the fluid flow. It is to be noted that Figure 6 is computed at $\hbar=-0.01$ and in Figure $7, \hbar=-0.05$ is used for stretching the sheet and $\hbar=-0.015,-0.004,-0.001,-0.0001$ are set for shrinking case along with the increasing value of $K$, respectively. Table 1 is evident that when sheet stretches, the local skin friction, local wall couple stress and heat transfer rate increase with the increasing concentration of microelements in the fluid flow while all of these physical quantities show reverse impact when the sheet shrinks as tabulated in Table 2. 


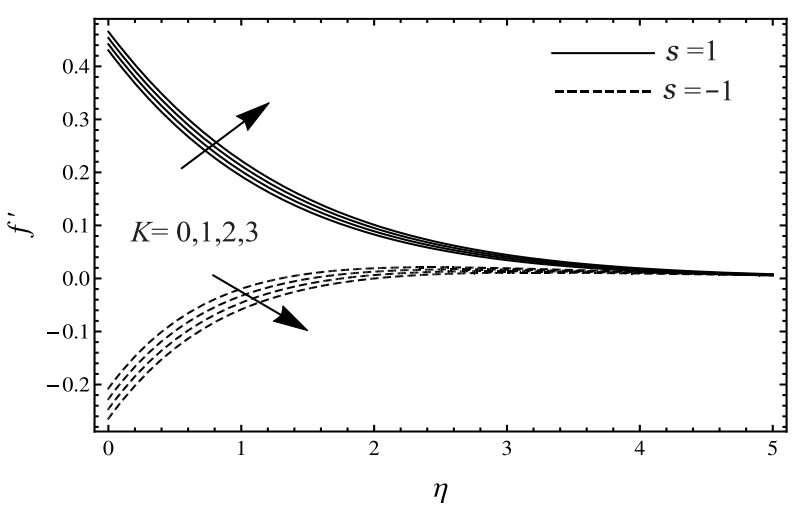

Figure 6. Influence of $K$ on $f^{\prime}$

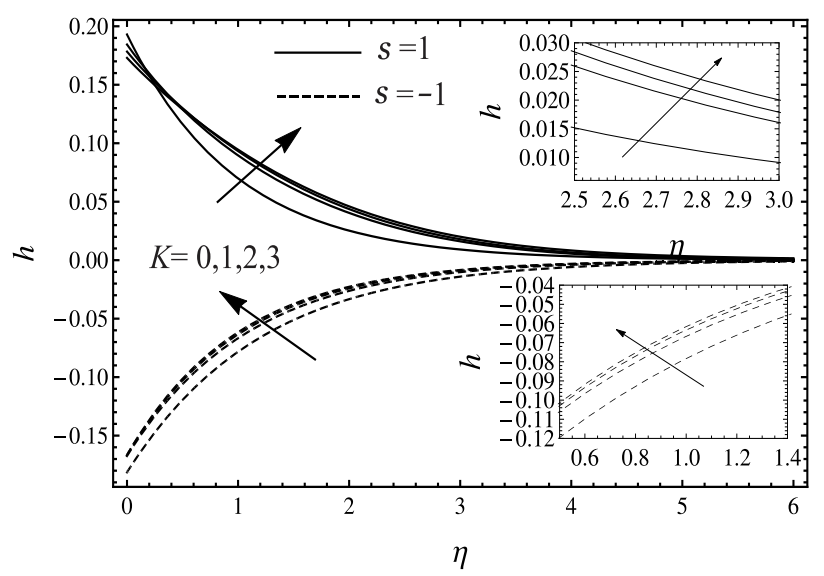

Figure 7. Influence of $K$ on $h$

The influence of the buoyancy parameter is depicted in Figure 8. It is observed that mixed convective micropolar fluid flow has higher velocity component as compared to the forced convective flow for both cases of the stretching and shrinking of the sheet. However, the velocity component is increasing with the increasing value of the buoyancy parameter when the sheet stretches or shrinks. Figure 8 is plotted at $\hbar=-0.01$ for $s=1$ and $\hbar=-0.005$ for $s=-1$. When the sheet stretches, an increase in the buoyancy parameter yields higher local skin friction and heat transfer rate in the micropolar fluid flow while it reduces the local wall couple stress as shown in Table 1. However, local skin friction and wall couple stress decrease and the local Nusselt number increases with the increasing value of the $\lambda$ for $s=-1$ as shown in Table 2 .

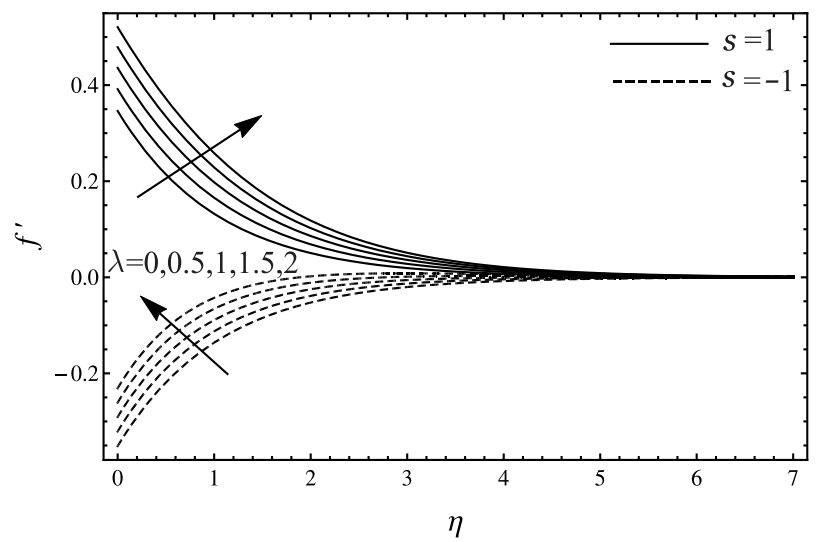

Figure 8. Influence of $\lambda$ on $f^{\prime}$

Figure 9 displays the impact of various values of the Prandtl number with the increasing value of the similarity variable $(\eta)$. It shows that either the sheet stretches or shrinks, a decrease in the thermal diffusivity or an increase in the momentum diffusivity, in other words, an increase in the Prandtl number causes to reduce the temperature of the micropolar fluid flow.

It is also noted that at the constant concentration of microelements at $(\eta=0)$, stretching sheet causes to reduce the $\approx 25 \%$ more temperature as compared to the shrinking of the sheet. However, the thickness of the thermal boundary layer is shorter in the shrinking case. Moreover, $\hbar=-0.01,-0.007$, $-0.006,-0.003,-0.0015,-0.0009,-0.0005,-0.0004,-0.0001$ and

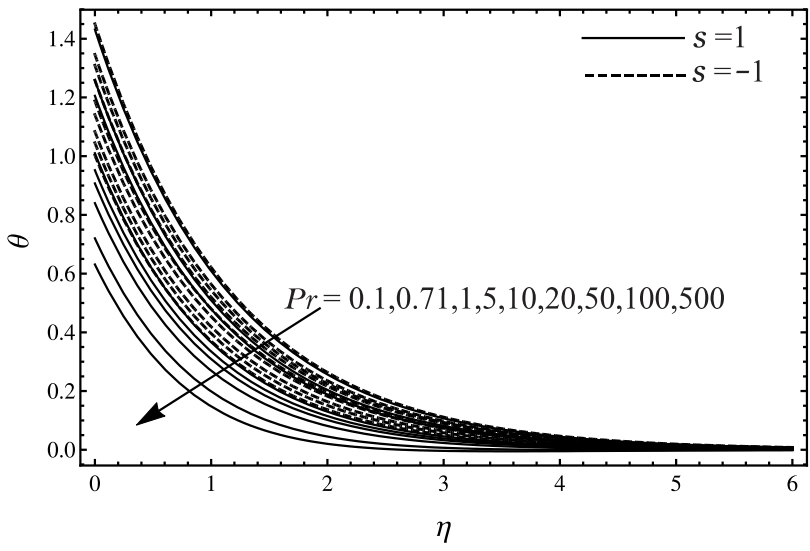

Figure 9. Influence of $\operatorname{Pr}$ on $\theta$

$\hbar=-0.01,-0.007,-0.006,-0.003,-0.0015,-0.0007,-0.0002$, $-0.00006,-0.000001$ are used for $s=1$ and $s=-1$ with the increasing value of the $\operatorname{Pr}$, respectively. When the momentum diffusivity is dominant on the thermal diffusivity, it is interesting to note that the heat transfer rate increases along with the local skin friction in both cases of the stretching and shrinking of the sheet as displayed in Tables 1-2. Furthermore, local wall couple stress increases for $s=1$, and it decreases for $s=-1$.

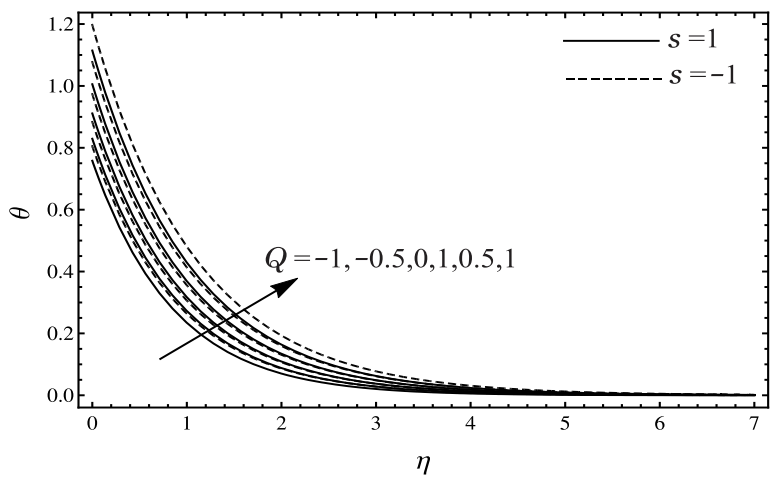

Figure 10. Influence of $Q$ on $\theta$

Figure 10 shows the influence of the heat generation $(>0)$ and absorption $(<0)$ parameter on the temperature profile at $\hbar=-0.001$. It is observed that an increase in the heat generation parameter results in an increase in the thermal boundary layer thickness which shows a rise in the temperature of the micropolar fluid flow and on the other hand, temperature reduces when heat absorption parameter increases. 
This flow behaviour is noticed when the sheet stretches or shrinks. An increase in the heat generation parameter yields a decrease in the local skin friction and contrastively, reduces the heat transfer rate. In addition, the local wall couple stress decreases. Furthermore, increasing value of the heat absorption parameter produces an increase in the local skin friction, and local wall couple stress and adversely, rising the heat transfer rate. This flow behaviour is numerically reported in the Tables 1-2.

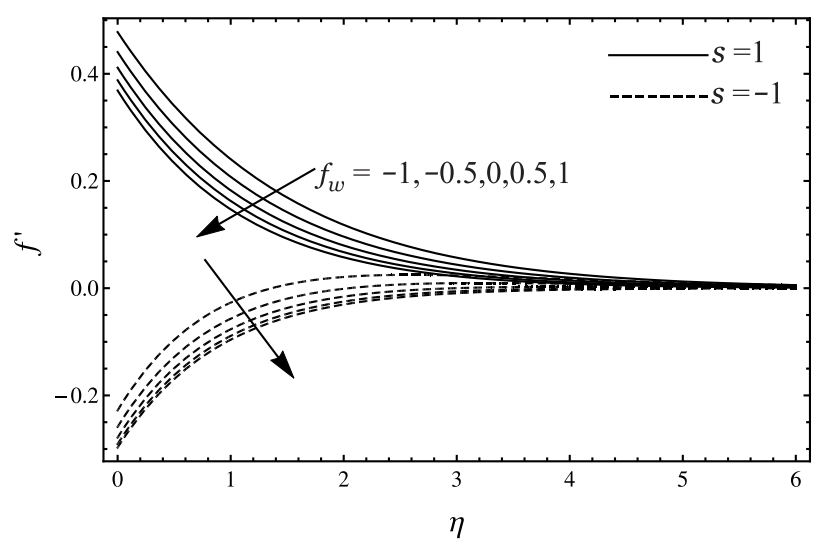

Figure 11. Influence of $f_{w}$ on $f^{\prime}$

Suction and injection effect on the velocity component is illustrated in Figure 11 at $\hbar=-0.005$. Obtained results show that when the sheet stretches or shrinks, higher the suction parameter lower the velocity component and higher the injection parameter, higher the velocity component of the micropolar fluid flow. It is seen from the Tables 3-4, local skin friction, local wall couple stress and local Nusselt number increase as the suction parameter increases, on the other hand, these physical quantities decrease with the increasing value of the injection parameter.

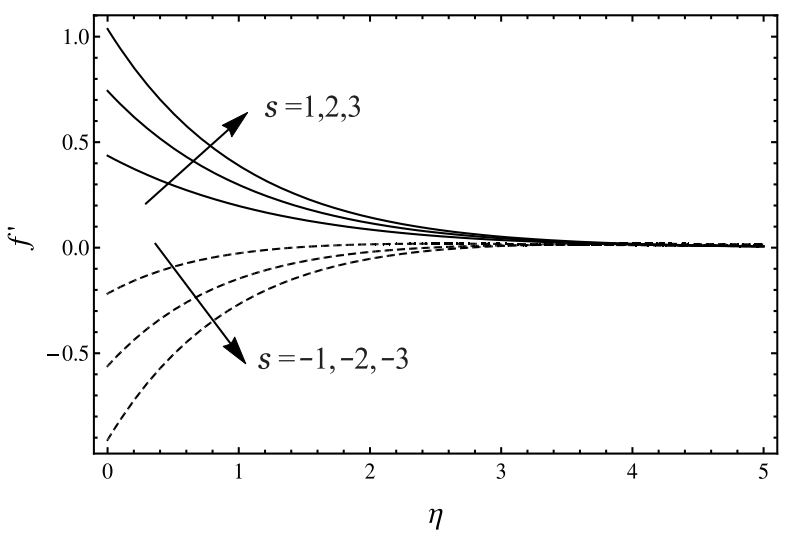

Figure 12. Influence of $s$ on $f^{\prime}$

An increase in the stretching parameter leads to an increase in the velocity component and an increase in the shrinking parameter results in a lower velocity component as sketched in Figure 12 at $\hbar=-0.01$. The impacts of the first order $(\alpha)$ and second order $(\beta)$ slip flow parameters on the velocity component are plotted in the Figures 13-14. It can be seen that increasing value of the slip flow parameters reduces the velocity component when the sheet stretches and in the shrinking case, velocity component shows reverse behaviour. In Figures 13-14, all curves are plotted at $\hbar=-0.01$ and $\hbar=-0.005$ is used for all values of $\beta$ for $s=-1$. From the
Tables 3-4, it is depicted that the increasing value of both slip flow parameters causes to reduce the local skin friction, local wall couple stress and local Nusselt number for $s=1$, a reverse phenomenon is observed for $s=-1$.

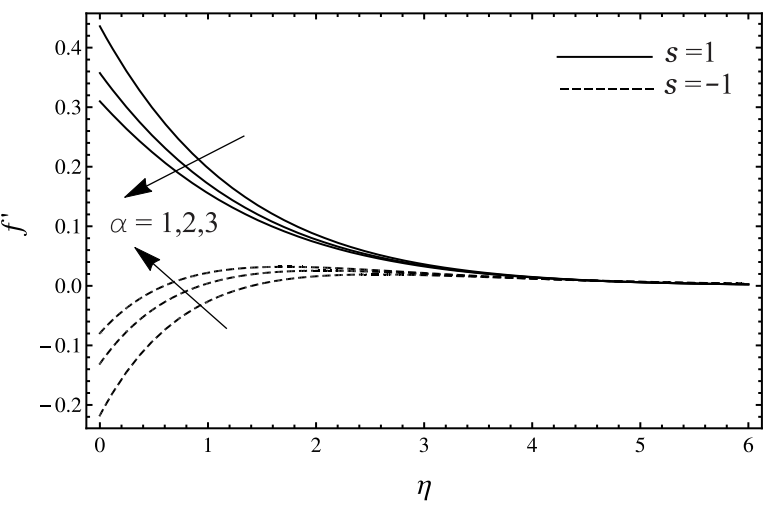

Figure 13. Influence of $\alpha$ on $f^{\prime}$

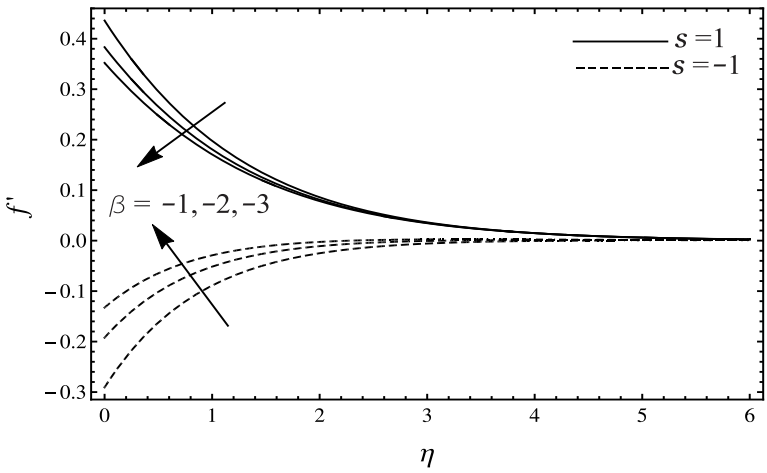

Figure 14. Influence of $\beta$ on $f^{\prime}$

An increase in the Newtonian heating parameter produces a higher thermal boundary layer thickness which results in a higher temperature of the micropolar fluid flow with the stretching or shrinking the sheet at $\hbar=-0.005$ as illustrated in Figure 15. It is observed from the sketch; shrinking sheet rises the $\approx 165 \%$ more temperature of the micropolar fluid flow as compared to the stretching of the sheet at $(\eta=0)$. This increase in temperature reinforces the heat transfer rate from the bonding surface of the micropolar fluid flow. However, local skin friction and local wall couple stress increase with stretching the sheet and decrease with shrinking the sheet as shown in Tables $3-4$.

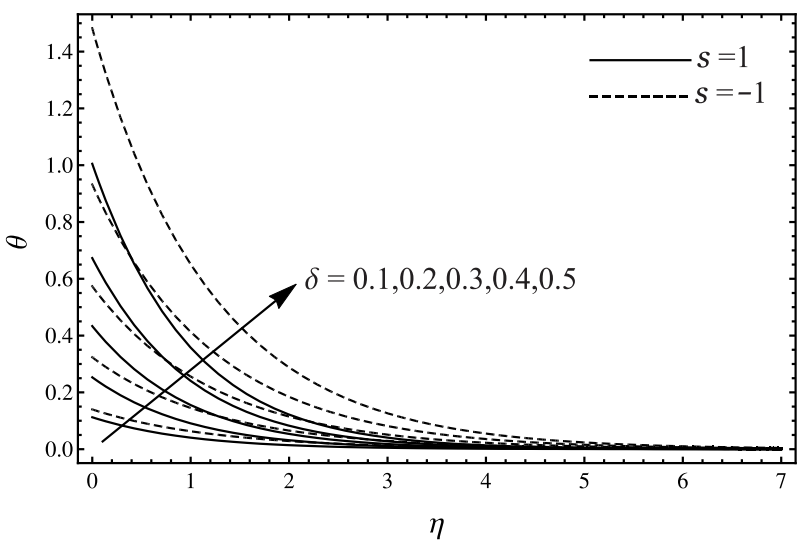

Figure 15. Influence of $\delta$ on $\theta$ 
Table 1. Influence of physical quantities for stretching sheet $(s=1)$

\begin{tabular}{cccccccccc}
\hline$K$ & $\lambda$ & $\operatorname{Pr}$ & $Q$ & $C_{f x} \mathrm{Re}_{x}^{1 / 2}$ & $\hbar$ & $M_{x} \mathrm{Re}_{x}$ & $\hbar$ & $N u_{x} \mathrm{Re}_{x}^{-1 / 2}$ & $\hbar$ \\
\hline 0.0 & 1.0 & 5 & 0.5 & 0.334177 & -0.01 & 0.164332 & -0.01 & 1.02377 & -0.01 \\
1.0 & & & & 0.499265 & -0.01 & 0.216982 & -0.01 & 1.03024 & -0.01 \\
2.0 & & & & 0.663223 & -0.01 & 0.260292 & -0.01 & 1.03686 & -0.01 \\
3.0 & & & & 0.82599 & -0.01 & 0.296952 & -0.01 & 1.0436 & -0.01 \\
0.5 & 0 & & & 0.416618 & -0.01 & 0.194074 & -0.01 & 0.986943 & -0.01 \\
& 0.5 & & & 0.416762 & -0.014 & 0.19298 & -0.01 & 1.00647 & -0.01 \\
& 1 & & & 0.416863 & -0.01 & 0.192078 & -0.01 & 1.02699 & -0.01 \\
& 1.5 & & & 0.417499 & -0.01 & 0.191363 & -0.01 & 1.04855 & -0.01 \\
& 2 & & & 0.418466 & -0.01 & 0.190831 & -0.01 & 1.07124 & -0.01 \\
& 1 & 0.1 & & 0.411391 & -0.01 & 0.189034 & -0.01 & 0.849324 & -0.01 \\
& 0.71 & & 0.414399 & -0.007 & 0.195391 & -0.007 & 0.897439 & -0.007 \\
& 1 & & 0.415103 & -0.006 & 0.197387 & -0.006 & 0.914727 & -0.006 \\
& 5 & & 0.416614 & -0.003 & 0.2031650 & -0.003 & 0.995062 & -0.003 \\
& 10 & & 0.416704 & -0.001 & 0.205789 & -0.0015 & 1.02511 & -0.0015 \\
& 20 & & 0.416795 & -0.001 & 0.206829 & -0.0009 & 1.05115 & -0.0009 \\
& 50 & & 0.417069 & -0.001 & 0.207517 & -0.0005 & 1.09526 & -0.0005 \\
& 100 & & 0.417501 & -0.001 & 0.207708 & -0.0004 & 1.27744 & -0.0005 \\
& 500 & & 0.418479 & -0.0014 & 0.208182 & -0.0001 & 1.29224 & -0.0001 \\
& 5 & 1.0 & 0.399504 & -0.01 & 0.182218 & -0.01 & 0.948628 & -0.001 \\
& & 0.5 & 0.416863 & -0.01 & 0.192078 & -0.01 & 0.997226 & -0.001 \\
& & 0.0 & 0.422514 & -0.01 & 0.195484 & -0.01 & 1.04874 & -0.001 \\
& & -0.5 & 0.424472 & -0.01 & 0.196784 & -0.01 & 1.10293 & -0.001 \\
& & -1.0 & 0.425194 & -0.01 & 0.197342 & -0.01 & 1.1595 & -0.001 \\
\hline
\end{tabular}

Table 2. Influence of physical quantities for shrinking sheet $(s=-1)$

\begin{tabular}{cccccccccc}
\hline$K$ & $\lambda$ & $\operatorname{Pr}$ & $Q$ & $C_{f x} \mathrm{Re}_{x}^{1 / 2}$ & $\hbar$ & $M_{x} \mathrm{Re}_{x}$ & $\hbar$ & $N u_{x} \mathrm{Re}_{x}^{-1 / 2}$ & $\hbar$ \\
\hline 0.0 & 1.0 & 5 & 0.5 & -0.344184 & -0.01 & -0.148705 & -0.01 & 0.719818 & -0.01 \\
1.0 & & & & -0.518381 & -0.01 & -0.221603 & -0.01 & 0.717166 & -0.01 \\
2.0 & & & & -0.6933 & -0.01 & -0.289342 & -0.01 & 0.714596 & -0.01 \\
3.0 & & & & -0.868475 & -0.01 & -0.351286 & -0.01 & 0.712104 & -0.01 \\
0.5 & 0 & & & -0.416061 & -0.01 & -0.168036 & -0.01 & 0.700696 & -0.01 \\
& 0.5 & & & -0.423981 & -0.01 & -0.177102 & -0.01 & 0.709329 & -0.01 \\
& 1 & & & -0.431161 & -0.01 & -0.18571 & -0.01 & 0.718481 & -0.01 \\
& 1.5 & & & -0.437622 & -0.01 & -0.193871 & -0.01 & 0.728196 & -0.01 \\
& 2 & & & -0.44338 & -0.01 & -0.201594 & -0.01 & 0.73852 & -0.01 \\
& 1 & 0.1 & & -0.423092 & -0.01 & -0.180243 & -0.01 & 0.844706 & -0.01 \\
& 0.71 & & -0.420096 & -0.007 & -0.185385 & -0.007 & 0.871355 & -0.007 \\
& 1 & & -0.419283 & -0.006 & -0.187689 & -0.006 & 0.881855 & -0.006 \\
& 5 & & -0.417694 & -0.003 & -0.196629 & -0.003 & 0.897228 & -0.003 \\
& 10 & & -0.417033 & -0.0015 & -0.202079 & -0.0015 & 0.920888 & -0.0015 \\
& 20 & & -0.416793 & -0.0007 & -0.20531 & -0.0007 & 0.938033 & -0.0007 \\
& 50 & & -0.416688 & -0.0002 & -0.207447 & -0.0002 & 0.961458 & -0.0002 \\
& 100 & & -0.416671 & -0.00007 & -0.208065 & -0.00006 & 0.9783 & -0.00006 \\
& 500 & & -0.416667 & -0.000001 & -0.208329 & -0.000001 & 0.998309 & -0.000001 \\
& 5 & 1.0 & -0.474301 & -0.01 & -0.214164 & -0.01 & 0.91759 & -0.001 \\
& & 0.5 & -0.431161 & -0.01 & -0.18571 & -0.01 & 0.964104 & -0.001 \\
& & 0.0 & -0.416723 & -0.01 & -0.175265 & -0.01 & 1.01365 & -0.001 \\
& & -0.5 & -0.411559 & -0.01 & -0.171018 & -0.01 & 1.066050 & -0.001 \\
& & -1.0 & -0.409513 & -0.01 & -0.169035 & -0.01 & 1.12102 & -0.001 \\
\hline
\end{tabular}

Table 3. Influence of physical quantities for stretching sheet $(s=1)$

\begin{tabular}{cccccccccc}
\hline$f_{w}$ & $\alpha$ & $\beta$ & $\delta$ & $C_{f x} \operatorname{Re}_{x}^{1 / 2}$ & $\hbar$ & $M_{x} \operatorname{Re}_{x}$ & $\hbar$ & $N u_{x} \operatorname{Re}_{x}^{-1 / 2}$ & $\hbar$ \\
\hline 1.0 & 1 & -1 & 0.5 & 0.423989 & -0.01 & 0.21345 & -0.01 & 1.05015 & -0.001 \\
0.5 & & & & 0.416863 & -0.01 & 0.192078 & -0.01 & 0.997226 & -0.001 \\
0.0 & & & & 0.400699 & -0.01 & 0.166242 & -0.01 & 0.949626 & -0.001 \\
-0.5 & & & & 0.366701 & -0.01 & 0.131686 & -0.01 & 0.906789 & -0.001 \\
-1.0 & & & & 0.297985 & -0.01 & 0.0802991 & -0.01 & 0.868219 & -0.001 \\
0.5 & 1 & & & 0.416863 & -0.01 & 0.192078 & -0.01 & 1.02699 & -0.01 \\
& 2 & & & 0.311641 & -0.01 & 0.140045 & -0.01 & 0.976273 & -0.01 \\
& 3 & & & 0.248795 & -0.01 & 0.109639 & -0.01 & 0.947576 & -0.01 \\
& 1 & -1 & & 0.416863 & -0.01 & 0.192078 & -0.01 & 1.02699 & -0.01 \\
& -2 & & 0.344601 & -0.01 & 0.156269 & -0.01 & 0.983415 & -0.01
\end{tabular}




\begin{tabular}{cccccccc}
-3 & & 0.302009 & -0.01 & 0.135619 & -0.01 & 0.958261 & -0.01 \\
-1 & 0.1 & 0.416665 & -0.001 & 0.193816 & -0.01 & 0.989341 & -0.005 \\
& 0.2 & 0.416664 & -0.001 & 0.193506 & -0.01 & 0.990621 & -0.005 \\
& 0.3 & 0.416663 & -0.001 & 0.193128 & -0.01 & 0.992267 & -0.005 \\
& 0.4 & 0.416661 & -0.001 & 0.19266 & -0.01 & 0.994465 & -0.005 \\
& 0.5 & 0.416659 & -0.001 & 0.192078 & -0.01 & 0.997557 & -0.005 \\
\hline
\end{tabular}

Table 4. Influence of physical quantities for stretching sheet $(s=-1)$

\begin{tabular}{cccccccccc}
\hline$f_{w}$ & $\alpha$ & $\beta$ & $\delta$ & $C_{f x} \operatorname{Re}_{x}^{1 / 2}$ & $\hbar$ & $M_{x} \operatorname{Re}_{x}$ & $\hbar$ & $N u_{x} \operatorname{Re}_{x}^{-1 / 2}$ & $\hbar$ \\
\hline 1.0 & 1 & -1 & 0.5 & -0.416111 & -0.01 & -0.18238 & -0.01 & 1.01385 & -0.001 \\
0.5 & & & & -0.431161 & -0.01 & -0.18571 & -0.01 & 0.964104 & -0.001 \\
0.0 & & & & -0.463233 & -0.01 & -0.188376 & -0.03 & 0.919418 & -0.001 \\
-0.5 & & & & -0.529064 & -0.01 & -0.210515 & -0.01 & 0.879249 & -0.001 \\
-1.0 & & & & -0.660126 & -0.01 & -0.269144 & -0.01 & 0.843118 & -0.001 \\
0.5 & 1 & & & -0.431161 & -0.01 & -0.18571 & -0.01 & 0.718481 & -0.01 \\
& 2 & & & -0.321567 & -0.01 & -0.142255 & -0.01 & 0.745677 & -0.01 \\
& 3 & & & -0.256442 & -0.01 & -0.115679 & -0.01 & 0.76341 & -0.01 \\
& 1 & -1 & & -0.431161 & -0.01 & -0.18571 & -0.01 & 0.718481 & -0.01 \\
& -2 & & -0.290721 & -0.01 & -0.128278 & -0.01 & 0.748154 & -0.01 \\
& -3 & & -0.205674 & -0.01 & -0.092495 & -0.01 & 0.768015 & -0.01 \\
& -1 & 0.1 & -0.417693 & -0.01 & -0.169863 & -0.01 & 0.814081 & -0.005 \\
& & 0.2 & -0.419752 & -0.01 & -0.172195 & -0.01 & 0.818069 & -0.005 \\
& & 0.3 & -0.422429 & -0.01 & -0.175274 & -0.01 & 0.82299 & -0.005 \\
& & 0.4 & -0.426042 & -0.01 & -0.179516 & -0.01 & 0.829213 & -0.005 \\
& & 0.5 & -0.431161 & -0.01 & -0.18571 & -0.01 & 0.837334 & -0.005 \\
\hline
\end{tabular}

\section{CONCLUSIONS}

In this investigation, heat generation/absorption effect on the mixed convective micropolar fluid flow over a permeable stretching/shrinking sheet has been analysed with the second order slip flow model. Newtonian heating is considered for this flow phenomenon. The transformed set of ordinary differential equations is solved by using an analytical method named as Homotopy Analysis Method (HAM). The current results are validated with the already published numerical and analytical results in an excellent manner. The following conclusions can be concluded from the present study,

- When sheet stretches or shrinks with the constant concentration of the microelements, higher the heat generation parameter; higher the thermal boundary layer thickness and on the other hand, higher the heat absorption parameter; lower the thermal boundary layer thickness.

- While analysing the Prandtl number impact, it is observed that an increase in the momentum diffusivity produces a fall in the temperature of the micropolar fluid flow and oppositely, it enhances the heat transfer rate.

- However, slip flow parameters ( $\alpha$ and $\beta$ ) show an exact correspondence with their definition in this fluid flow. When stretching of the sheet takes place, velocity component decreases with the increasing value of the slip flow parameters, and when the sheet shrinks, local skin friction enhances in fluid flow.

- Furthermore, the higher value of the Newtonian heating parameter rises the temperature of the micropolar fluid flow, which also results in a higher heat transfer rate.

\section{ACKNOWLEDGMENT}

This research is financially supported by, Spk: 319299-Ver 1, 6353 Curtin University-SCI \& ENG, Department of Mathematics and Statistics/CIPRS. Moreover, there is no conflict of interest in this presented research, and the author acknowledges the review of the Professor Asterios Pantokratoras from the Democritus University of Thrace, Xanthi, Thrace, Greece.

\section{REFERENCES}

[1] Eringen AC. (1964). Simple microfluids. International Journal of Engineering Science 2: 205-217. https://doi.org/10.1016/0020-7225(64)90005-9

[2] Eringen AC. (1965). Theory of micropolar fluids. Purdue University Lafayette in School of Aeronautics and Astronautics, USA, Technical Report 27.

[3] Eringen AC. (1972). Theory of thermomicrofluids. Journal of Mathematical Analysis and Applications 38: 480-496. https://doi.org/10.1016/0022 -247X(72)901060

[4] Eringen AC. (2001). Microcontinuum Field Theories: II Fluent Media, Springer-Verlag, New York, Inc.

[5] Lukaszewicz G. (1999). Micropolar Fluids: Theory and Applications. Springer Science \& Business Media, New York. https://doi.org/10.1007/978-1-4612-0641-5

[6] Kelson NA, Farrell TW. (2001). Micropolar flow over a porous stretching sheet with strong suction or injection. International Communications of Heat and Mass Transfer 28(4): 479-488. https://doi.org/10.1016/S07351933(01)00252-4

[7] Kelson NA, Desseaux A. (2001). Effect of surface conditions on flow of a micropolar fluid driven by a porous stretching sheet. International Journal of 
Engineering Science 39(16): 1881-1897. https://doi.org/10.1016/S0020-7225(01)00026-X

[8] Bhargava R, Kumar L, Takhar HS. (2003). Finite element solution of mixed convection micropolar flow driven by a porous stretching sheet. International Journal of Engineering Science 41(18): 2161-2178. https://doi.org/10.1016/S0020-7225(03)00209-X

[9] Ahmed SE, Ahmed KH, Mohammed HA, Sivasankaran S. (2014). Boundary layer flow and heat transfer due to permeable stretching tube in the presence of heat source/sink utilizing nanofluids. Applied Mathematics and Computation 238: 149-162.

https://doi.org/10.1016/j.amc.2014.03.106

[10] Chand R, Rana GC, Hussein AK. (2015). Effect of suspended particles on the onset of thermal convection in a nanofluid layer for more realistic boundary conditions. International Journal of Fluid Mechanics Research 42: 375-390.

https://doi.org/10.1615/InterJFluidMechRes.v42.i5.10

[11] Turkyilmazoglu M. (2014). A note on micropolar fluid flow and heat transfer over a porous shrinking sheet. International Journal of Heat and Mass Transfer 72: 388391.

https://doi.org/10.1016/j.ijheatmasstransfer.2014.01.039

[12] Turkyilmazoglu M. (2016). Flow of a micropolar fluid due to a porous stretching sheet and heat transfer. International Journal of Non-Linear Mechanics 83: 5964. https://doi.org/10.1016/j.ijnonlinmec.2016.04.004

[13] Pal D, Mandal G. (2017). Thermal radiation and MHD effects on boundary layer flow of micropolar nanofluid past a stretching sheet with non-uniform heat source/sink International Journal of Mechanical Sciences 126: 308318. https://doi.org/10.1016/j.ijmecsci.2016.12.023

[14] Wu L. (2008). A slip model for rarefied gas flows at arbitrary Knudsen number. Applied Physics Letters 93(25): 253103. https://doi.org/10.1063/1.3052923

[15] Burgdorfer A. (1959). The influence of the molecular mean free path on the performance of hydrodynamic gas lubricated bearings. ASME Journal of Basic Engineering 81: 94-100.

[16] Hsia YT, Domoto GA. (1983). An experimental investigation of molecular rarefaction effects in gas lubricated bearings at ultra-low clearances. Journal of Lubrication Technology 105(1): 120-129. https://doi.org/10.1115/1.3254526

[17] Mitsuya Y. (1993). Modified Reynolds equation for ultra-thin film gas lubrication using 1.5-order slip-flow model and considering surface accommodation coefficient. Journal of Tribology 115(2): 289-289. https://doi.org/10.1115/1.2921004

[18] Fukui S, Kaneko R. (1990). A database for interpolation of Poiseuille flow rates for high Knudsen number lubrication problems. Journal of Tribology 112(1): 7883. https://doi.org/10.1115/1.2920234

[19] Fang T, Yao S, Zhang J, Aziz A. (2010). Viscous flow over a shrinking sheet with a second order slip flow model. Communications in Nonlinear Science and Numerical Simulation 15(7): 1831-1842. https://doi.org/10.1016/j.cnsns.2009.07.017

[20] Nandeppanavar MM, Vajravelu K, Abel MS, Siddalingappa MN. (2012). Second order slip flow and heat transfer over a stretching sheet with non-linear Navier boundary condition International Journal of

Thermal Sciences 58: 143-150.

https://doi.org/10.1016/j.ijthermalsci.2012.02.019

[21] Singh G, Chamkha AJ. (2013). Dual solutions for second-order slip flow and heat transfer on a vertical permeable shrinking sheet. Ain Shams Engineering Journal 4(4): 911-917. https://doi.org/10.1016/j.asej.2013.02.006

[22] Rosca NC, Pop I. (2013). Mixed convection stagnation point flow past a vertical flat plate with a second order slip: heat flux case. International Journal of Heat and Mass Transfer 65: 102-109. https://doi.org/10.1016/j.ijheatmasstransfer.2013.05.061

[23] Rosca NC, Pop I. (2014). Boundary layer flow past a permeable shrinking sheet in a micropolar fluid with a second order slip flow model. European Journal of Mechanics-B/Fluids 48: 115-122. https://doi.org/10.1016/j.euromechflu.2014.05.004

[24] Sharma R, Ishak A, Pop I. (2016). Stagnation point flow of a micropolar fluid over a stretching/ shrinking sheet with second-order velocity slip. Journal of Aerospace Engineering 29(5): 04016025. https://doi.org/10.1061/(ASCE)AS.1943-5525.0000616

[25] Ibrahim W. (2017). MHD boundary layer flow and heat transfer of micropolar fluid past a stretching sheet with second-order slip. Journal of the Brazilian Society of Mechanical Sciences and Engineering 39(3): 791-799. https://doi.org/10.1007/s40430-016-0621-8

[26] Kamran, M., Wiwatanapataphee B. (2018). Chemical reaction and Newtonian heating effects on steady convection flow of a micropolar fluid with second order slip at the boundary. European Journal of MechanicsB/Fluids 71: 138-150. https://doi.org/10.1016/j.euromechflu.2018.04.005

[27] Merkin JH. (1994). Natural-convection boundary-layer flow on a vertical surface with Newtonian heating. International Journal of Heat and Fluid Flow 15(5): 392 398. https://doi.org/10.1016/0142-727X(94)90053-1

[28] Ahmadi G. (1976). Self-similar solution of incompressible micropolar boundary layer flow over a semi-infinite plate. International Journal of Engineering Science 14(7): 639-646. https://doi.org/10.1016/00207225(76)90006-9

[29] Liao S. (2003). Beyond perturbation: Introduction to the Homotopy Analysis Method. CRC Press. https://doi.org/10.1201/9780203491164

[30] Narahari M, Kamran M. (2016). MHD natural convection flow past an impulsively started infinite vertical porous plate with Newtonian heating in the presence of radiation. International Journal of Numerical Methods for Heat \& Fluid Flow 26(6): 1932-1953. https://doi.org/10.1108/HFF-03-2015-0086

\section{NOMENCLATURE}

$A$

B

$c_{p}$

$d$

$f$

$f_{w}$ constant coefficient for mean free path, $m$ square of constant coefficient for mean free path, $m^{2}$ specific heat at constant pressure, $J \cdot K^{-1} \cdot K^{-1}$ mean free path, $m$ dimensionless velocity dimensionless suction/injuction parameter 
gravitational acceleration, $m \cdot s^{-2}$

dimensionless local Grashof number

surface heat transfer coefficient, $W \cdot m^{-2} \cdot K^{-1}$

dimensionless micro-rotational velocity

microinertia per unit mass, $\mathrm{m}^{2}$

dimensionless material parameter

thermal conductivity, $W \cdot m^{-1} \cdot K^{-1}$

Knudsen number

constant

dimensionless Prandtl number

dimensionless heat generation/absorption parameter

volumetric rate of heat generation, $W \cdot m^{-3} \cdot K^{-1}$

dimensionless local Reynolds number

stretching/ shrinking constant

fluid temperature, $K$

horizontal velocity component, $m \cdot s^{-1}$

vertical velocity component, $m \cdot s^{-1}$

suction/injection velocity, $m . s^{-1}$

horizontal Direction, $m$

transverse directions, $m$

\section{Greek symbols}

$\alpha \quad$ dimensionless first order slip flow parameter

$\beta \quad$ dimensionless second order slip flow parameter

$\beta_{T} \quad$ coefficient of thermal expansion, $K^{-1}$

$\gamma \quad$ spin gradient viscosity coefficient, $K g . \mathrm{m} . \mathrm{s}^{-1}$

$\delta \quad$ dimensionless Newtonian heating parameter

$\in \quad$ momentum accommodation coefficient

$\theta \quad$ dimensionless temperature

$\lambda$ mimensionless Buoyancy parameter

$\mu \quad$ dynamic viscosity, N.s. $m^{-2}$

$v \quad$ kinematic viscosity, $m^{2} \cdot s^{-1}$

$\psi \quad$ stream function, $m^{2} \cdot s^{-1}$

$\rho \quad$ fluid density, $\mathrm{Kg} \cdot \mathrm{m}^{-3}$

$\chi \quad$ micro-rotation viscosity, $K g \cdot \mathrm{m}^{-1} \cdot \mathrm{s}^{-1}$

$\omega \quad$ micro-rotational velocity (cartesian), $m \cdot s^{-1}$

\section{Subscripts}

$\begin{array}{ll}p & \text { constant pressure } \\ \infty & \text { free stream }\end{array}$

